The Astrophysical Journal, 194:L97-L101, 1974 December 1

(c) 1974. The American Astronomical Society. All rights reserved. Printed in U.S.A.

\title{
RADIATIVE TRAPPING AND POPULATION INVERSIONS OF THE SiO MASERS*
}

\author{
JoHN KWaN \\ Institute for Advanced Study \\ AND \\ Nick Scoville \\ Owens Valley Radio Observatory, and California Institute of Technology \\ Received 1974 August 14; revised 1974 September 18
}

\section{ABSTRACT}

Radiative trapping in the $v=0 \rightarrow 1$ vibrational transitions decreases the rate of de-excitation of a $v=1$ level as its rotational quantum number increases. This effect readily leads to population inversions of the $v=1$ rotational transitions if population is transferred from $v=0$ into $v=1$ by collisions or by an indirect radiative route, as, for example, through absorptions from $v=0$ into $v=2$ and the subsequent spontaneous cascade from $v=2$ to $v=1$. Numerical calculations which model the $\mathrm{SiO}$ masers discovered toward stars give photon emission rates in the $J=1 \rightarrow$ $0, J=2 \rightarrow 1$, and $J=3 \rightarrow 2$ transitions which are comparable and substantial, $\sim 3 \times 10^{43} \mathrm{~s}^{-1}$.

Subject headings: masers - radiative transfer

\section{INTRODUCTION}

Rotational lines from the first excited vibrational state of $\mathrm{SiO}$ were recently discovered toward Orion and several infrared stars (Snyder and Buhl 1974; Davis et al. 1974). The $v=1, J=3 \rightarrow 2, J=2 \rightarrow 1$, and $J=1 \rightarrow 0$ lines are all observed, leaving little doubt about their identification with $\mathrm{SiO}$. The unexpectedly high intensities of the lines and the structures of their profiles, in contrast to what have been observed of rotational lines of the ground vibrational state, argue strongly for maser action in those lines.

An estimate of the size of the maser region is prerequisite to limiting the range of physical conditions under which we may search for a viable inversion mechanism. We estimate the size from the observed flux density, $S_{\nu}$ (ergs $\mathrm{s}^{-1} \mathrm{~cm}^{-2} \mathrm{~Hz}^{-1}$ ), and the maser intensity at the surface of the cloud, $I\left(\operatorname{ergs~s}^{-1} \mathrm{~cm}^{-2}\right.$ $\mathrm{Hz}^{-1} \mathrm{sr}^{-1}$ ), through the following relation:

$$
I \Omega r^{2} / d^{2}=S_{\nu}
$$

where $d$ is the distance to the maser cloud, and $r$ its radius (or length if the maser is tubular rather than spherical). The solid angle $\Omega$ into which the maser radiation is beamed relates the apparent size to the physical size of the cloud. Noting that the stimulated emission rate $W$ is $B I \Omega / 4 \pi$, we rewrite equation (1) in the form

$$
r=d\left(\frac{S_{\nu} B}{4 \pi W}\right)^{1 / 2}
$$

and an estimate of the size is obtained if $W$ is known. The latter rate is approximately limited by the rate $\Gamma\left(\mathrm{s}^{-1}\right)$ at which population of a maser level is transferred to other levels by means other than maser

* This research is sponsored by the National Science Foundation grant GP-40768X (J.K.) and GP-30400-X5 (N.S.) and by the Office of Naval Research under contract N0014-67-A-0094-0019(N.S.). emission or absorption. When $W>\Gamma$, the maser saturates and the stimulated emission rate then increases only linearly with increasing path length $r$. Observations at Orion suggest that the $\mathrm{SiO}$ masers are saturated, but are not heavily so. On the one hand, the observation of both $v=1, J=3 \rightarrow 2$ and $J=2 \rightarrow 1$ lines and the broad line widths $\left(\sim 5 \mathrm{~km} \mathrm{~s}^{-1}\right)$ suggest saturation; on the other, the variation of the ratio of the $J=3 \rightarrow 2$ to the $J=2 \rightarrow 1$ intensity indicates that the saturation is probably mild. The size $r$ can therefore be obtained quite reliably from using an upper limit to $\Gamma$ and $W \sim \Gamma$.

The fastest rate $\Gamma$ of population transfer out of a maser level to other SiO levels is the rate of spontaneous decay to the ground vibrational state. Assuming a ratio of the $v=1 \rightarrow 0$ vibrational transition strength to the pure rotational transition strength of $10^{-3}$ and neglecting opacity effects, we estimate an upper limit to $\Gamma$ of $\sim 5 \mathrm{~s}^{-1}$. The observed flux density of $\sim 10^{3}$ fu of the $J=2 \rightarrow 1$ line in Orion then implies

$$
r=1.2 \times 10^{15} \mathrm{~cm} \frac{d}{500 \mathrm{pc}}\left(\frac{S_{\nu}}{10^{3} \mathrm{fu}}\right)^{1 / 2}\left(\frac{5}{W}\right)^{1 / 2} .
$$

The $J=2 \rightarrow 1$ emission of the $\mathrm{SiO}$ sources discovered toward infrared stars are weaker or comparable to that observed at Orion (Davis et al. 1974). Because these stars are usually closer to us than Orion, the physical sizes of the $\mathrm{SiO}$ masers near them would be smaller.

\section{PUMP MECHANISM}

The pump mechanism we propose for the $v=1$ masers of $\mathrm{SiO}$ requires two conditions to be satisfied: first, that radiative de-excitation of $v=1$ to $v=0$ be more rapid than collisional de-excitation; and second, that the $v=0 \rightarrow 1$ vibrational transitions be optically thick. When these two conditions are met, the rate of 
depopulation of a $v=1$ rotational level is

$$
\begin{aligned}
\gamma & =\frac{A_{01}}{\tau_{01}}, & J=0, \\
& =\frac{A_{J, J-1}}{\tau_{J J-1}}+\frac{A_{J, J+1}}{\tau_{J, J+1}}, & J>0 .
\end{aligned}
$$

Here $A_{m, n}$ refers to the Einstein $A$ coefficient for the transition $v=1, J=m \rightarrow v=0, J=n$, and $\tau_{m, n}$ is the corresponding line opacity. Equations (4) can be rewritten, in terms of $A_{01} / \tau_{01}=p$, as

$$
\begin{array}{rlrl}
\gamma= & p, & J=0 \\
= & \frac{p}{2 J+1} & \\
& \times\left[\frac{n_{1}}{3}\left(\frac{2 J-1}{n_{J-1}}\right)+\frac{n_{1}}{3}\left(\frac{2 J+3}{n_{J+1}}\right)\right], & J>0 \\
\simeq \frac{2 p}{2 J+1}, & J>0 .
\end{array}
$$

The symbol $n_{J}$ denotes the population in the $v=0, J$ rotational level. In the last step of equations (5), we have assumed $\frac{1}{3} n_{1}\left[(2 J+1) / n_{J}\right] \approx 1$ for $J \geq 0$, as we expect that collisions among $v=0$ rotational levels would equilibrate the latter at the kinetic temperature which is much higher than the equivalent temperatures of the rotational energies, at least for $J \leq 6$. Relative to the de-excitation rate when the $v=0 \rightarrow 1$ vibrational transitions are thin, namely,

$$
\gamma=A_{01}, \quad J \geq 0,
$$

we readily observe that radiative trapping decreases the de-excitation rate as $J$, the rotational quantum number, increases. This effect arises when the $v=$ $0 \rightarrow 1$ transitions are thick because in each thick line the same number of photons escape and the depopulation of a $v=1$ level then depends on the number of routes available to it for de-excitation. Thus, the $v=1, J=1$ level has two routes for de-excitation whereas the $v=1, J=0$ level has only one; twice as much population is then removed from $J=1$ as is removed from $J=0$. The depopulation per sublevel, however, is smaller for $J=1$ than for $J=0$.

Of course, if the $v=1$ state is populated only by radiative absorptions from the $v=0$ state, there can be no inversions of the $v=1$ rotational levels, even if the vibrational transitions are thick, because then the population into $v=1$ shows exactly the same selectivity as the depopulation out of $v=1$. However, if population is transferred from $v=0$ to $v=1$ by collisions, or by an indirect radiative route, population inversions of the $v=1$ levels are possible. To illustrate the latter situation, we consider the radiative interactions among the three lowest vibrational states, and use a luminous infrared star as the radiation source. All collisions will be suppressed. We assume the $v=0 \rightarrow 1$ and the $v=$ $0 \rightarrow 2$ transitions thick, and the $v=1 \rightarrow 2$ transitions thin. We make two additional simplifications: first, that the $v=2$ state is populated mainly by radiative absorptions from $v=0$, and is de-excited mainly by spontaneous decays to $v=1$; and second, that the $v=0$ levels have equal populations per sublevel. We define

$$
\alpha(\nu)=\frac{I(\nu)}{h c}\left(\frac{\pi r_{*}{ }^{2}}{r^{2}}\right) \frac{\partial v}{\partial r},
$$

where $I$ is the stellar intensity, $r_{*}$ the stellar radius, $r$ the radius at the maser region, and $\partial v / \partial r$ the radial velocity gradient. The symbol $\alpha(\nu)$ denotes the number of photons that are absorbed per unit volume per second due to a thick line at frequency $\nu$. With the above approximations and simplifications, the populations in the $v=2$ and $v=1$ states, $n_{J}^{\prime \prime}$ and $n_{J}^{\prime}$ are

$$
\begin{aligned}
n_{J}{ }^{\prime \prime} & =\frac{\alpha\left(\nu_{02}\right)}{A}, \quad J=0: \\
n_{J}{ }^{\prime \prime} & =\frac{2 \alpha\left(\nu_{02}\right)}{A}, \quad J>0: \\
n_{0}{ }^{\prime} & =\frac{1}{q}\left[\alpha\left(\nu_{01}\right)+\frac{2}{3} \alpha\left(\nu_{02}\right)\right] ; \\
\frac{n_{1}{ }^{\prime}}{3} & =\frac{1}{q}\left[\alpha\left(\nu_{01}\right)+\frac{9}{10} \alpha\left(\nu_{02}\right)\right], \\
\frac{n_{2}^{\prime}}{5} & =\frac{1}{q}\left[\alpha\left(\nu_{01}\right)+\frac{23}{21} \alpha\left(\nu_{02}\right)\right], \\
\frac{n_{3}{ }^{\prime}}{7} & =\frac{1}{q}\left[\alpha\left(\nu_{01}\right)+\frac{47}{45} \alpha\left(\nu_{02}\right)\right] .
\end{aligned}
$$

Here $A$ represents the spontaneous de-excitation rate from each level of $v=2$ down to $v=1$, and $q=$ $\left(8 \pi \nu_{01}{ }^{3}\langle\partial v / \partial r\rangle\right) / n_{0} c^{3}$. The angular brackets around $\partial v / \partial r$ represent an average over all angles, and account for the fact that while the incident stellar radiation is absorbed primarily radially, spontaneous emission can occur in any one direction. The criterion for population inversion of the $v=1$ levels stated earlier, that the $v=0 \rightarrow 1$ transitions be thick, refers to the opacity for spontaneous emission.

We observe, from equations (8b), that population inversions for $J=2 \rightarrow 1$ and $J=1 \rightarrow 0$ are comparable and quite substantial. When both transitions are saturated, the number of $J=2 \rightarrow 1$ maser photons emitted to that of $J=1 \rightarrow 0$ emitted is 1.23 . There is no population inversion for $J=3 \rightarrow 2$ or higher rotational transitions. However, when $J=2 \rightarrow 1$ is saturated, the population in $J=2$ becomes smaller and inversion of $J=3 \rightarrow 2$ is then possible and quite strong, as solution of the rate equations including maser saturation will demonstrate.

The maser action in the $v=1$ rotational levels will be quenched when the $v=1 \rightarrow 2$ vibrational transitions become thick, as then the population from $v=2$ into $v=1$ shows the same selectivity as the depopulation out of $v=1$. This effect, together with collisional quenching, places an upper limit on the $\mathrm{SiO}$ and hydrogen densities. 
The above discussion can be generalized to obtain population inversions of the rotational transitions of any excited vibrational state. For example, population inversions of the $v=2$ levels would occur in the situation in which the $v=0 \rightarrow 2$ and $v=1 \rightarrow 2$ vibrational transitions are thick, but the $v=2 \rightarrow 3$ transitions are thin.

\section{SPECIFIC MODEL FOR STELLAR SIO SOURCES}

To demonstrate the proposed pumping mechanism, we present in this section numerical calculations which attempt to model the $\mathrm{SiO}$ sources discovered toward stars. For these sources a detailed model is possible, because the energy supply for the maser, the structure of the star, and the kinematics of the maser region are all better understood (Kwok, Gilman, and Woolf 1974; Elitzur, Goldreich, and Scoville 1974). It is very likely that the pumping of these sources is by radiation rather than by collisions. First, the number of stellar photons emitted at frequencies of the vibrational transitions $(8 \mu, 4 \mu$, etc.) is sufficient to account for the maser outputs. Second, if the excitation from $v=0$ to $v=1$ is due to collisions, then the subsequent escape of the $8 \mu$ photons would by itself lead to a rapid cooling of the gas. If the rate of production of $J=2 \rightarrow 1$ maser photons is $\sim 3 \times 10^{43} \mathrm{~s}^{-1}$, then the total rate of loss of $8-\mu$ photons must be $\sim 3 \times 10^{45} \mathrm{~s}^{-1}$. At the radius of $\sim 2 \times 10^{14} \mathrm{~cm}$ (cf. fig. 1) where the maser occurs, the thermal energy of the gas, with density $\sim 10^{9} \mathrm{~cm}^{-3}$ (corresponding to a mass outflow rate of $10^{-5} M_{\odot}$ $\mathrm{yr}^{-1}$ ) and kinetic temperature $\sim 300^{\circ} \mathrm{K}$, would be radiated away in $2 \times 10^{6} \mathrm{~s}$. This time is shorter than the time of gas-dust collisions which are probably the dominant processes of heating the gas. At 4-8 $\mu$, the radiation from circumstellar dust is weak compared with that from the star, and we shall use the dilute stellar radiation as the pump source.

We have studied numerically the excitation of 18 $\mathrm{SiO}$ vibration rotational levels, $v=0,1,2$ and $J \leq 5$. Because the masers occur in an expanding envelope about a central star, we utilize the escape-probability formulation of line transfer by Castor (1970) and Lucy (1971). The following parameters, adopted for the model calculations, are chosen to simulate the physical conditions of the maser region. The stellar luminosity, temperature, and radius are $10^{4} L_{\odot}, 2000^{\circ} \mathrm{K}$, and $5.8 \times$ $10^{13} \mathrm{~cm}$. The abundance ratio $[\mathrm{SiO}] /\left[\mathrm{H}_{2}\right]$ is $4 \times 10^{-5}$ (Dolan 1965; Vardya 1966), corresponding to twothirds of the silicon being in SiO. The vibrational line strengths for $\Delta v=1$ and $\Delta v=2$ transitions are $10^{-3}$ and $2 \times 10^{-4}$ that of pure rotational transitions. The expansion velocity varies with radius as $V=V_{0}(r)$ $\left.r_{0}\right)^{\epsilon}$, with $V_{0}=4 \mathrm{~km} \mathrm{~s}^{-1}, r_{0}=2 \times 10^{14} \mathrm{~cm}$ and, $\epsilon=$ 0.2 . The run of gas density with radius satisfies continuity of mass outflow and is normalized to give a specified rate of mass loss in the range $10^{-5}$ to $10^{-6} M_{\odot}$ $\mathrm{yr}^{-1}$.

In one model calculation we suppress all collisions except those among the rotational levels of the ground vibrational state. These collisions, within $v=0$, are specified to follow dipole selection rules $(\Delta J= \pm 1$, and a downward collisional cross-section of $4 \times 10^{-16}$ $\mathrm{cm}^{2}$ ), such that no maser action results from collisions among pure rotational levels (Goldsmith 1972). The results of this calculation are presented in figure 1 (dashed curves), where the emission rate of maser photons from each shell

$$
\frac{d N_{\nu}(r)}{d r}=4 \pi r^{2} W\left(n_{u}^{\prime}-n_{l}{ }^{\prime} \frac{g_{u}}{g_{l}}\right)
$$

is plotted against radius. The number of $4-\mu$ photons available per second in each thick line is also shown (dotted curve). The total emission rate of photons in each maser transition is equal to the integral area under each of the curves. For the three transitions $J=1 \rightarrow 0$, $J=2 \rightarrow 1$, and $J=3 \rightarrow 2$, they are respectively $2 \times 10^{43} \mathrm{~s}^{-1}, 4 \times 10^{43} \mathrm{~s}^{-1}$, and $2.7 \times 10^{43} \mathrm{~s}^{-1}$. The total $4-\mu$ flux available in one thick line is $\sim 10^{44} \mathrm{~s}^{-1}$. We believe this model provides a good example of the $\mathrm{SiO}$ masers.

The results of two other models are also presented in figure 1 (solid curves). These two models, with mass loss rates of $10^{-5}$ and $10^{-6} M_{\odot} \mathrm{yr}^{-1}$, differ from the previous one in that collisions within $v=0, v=1$, and $v=2$, as well as collisions between $\Delta v=1$ levels are all included. The gas kinetic temperature is assumed to vary inversely with radius from the star, being $2000^{\circ} \mathrm{K}$ at the stellar surface. This kinetic temperature is undoubtedly too high near the star, and because of the cooling argument presented earlier we do not believe these two models to be representative of the $\mathrm{SiO}$ masers. They are presented only to illustrate that, over the maser region found from the pure radiative model, collisions do not quench the inversions. Indeed, collisional excitations from $v=0$ to $v=1$ even increase the maser output above that given by pure radiative excitations. The collisional cross-sections we assume for $\Delta v=1$ transitions remain to be specified. The downward collisional cross-section from the $M$ rotational level of $v=1$ to the $J$ rotational level of $v=0$, for example, is $4 \times 10^{-16} \mathrm{~cm}^{2}[(2 J+1) /(6 M+3)]$ for $J=M$, $M-1$, or $M+1$, and is zero for other $J$ values.

Virtually all aspects of the pump mechanism discussed in $\S$ III are borne out by the detailed calculations. Most important, we find, as the star is approached from afar, that the inversion sets in immediately and strongly (about a 15\% inversion for $J=1 \rightarrow 0$ and $J=2 \rightarrow 1$ and $2 \%$ for $J=3 \rightarrow 2$ ) when the $v=1 \rightarrow 0$ transitions become thick to their spontaneous emission. Because $\epsilon<1$, these transitions are already thick to the stellar radiation. And, although the inversions commence at $1.1 \times 10^{15} \mathrm{~cm}$ in the two models with a massloss rate of $10^{-5} M_{\odot} \mathrm{yr}^{-1}$, the gains are insufficient to build up observable masers at that radius. This is because the lower SiO density and the more severe dilution of the radiation there do not provide sufficient population in $v=1$. Quenching of the masers will occur at some inner radius where the $\mathrm{H}_{2}$ density is high enough that collisional de-excitation of $v=1$ dominates over radiative de-excitation or where the $v=1 \rightarrow 2$ transitions become thick. Both of these effects occur at $10^{14} \mathrm{~cm}$ in the two high-density models; 


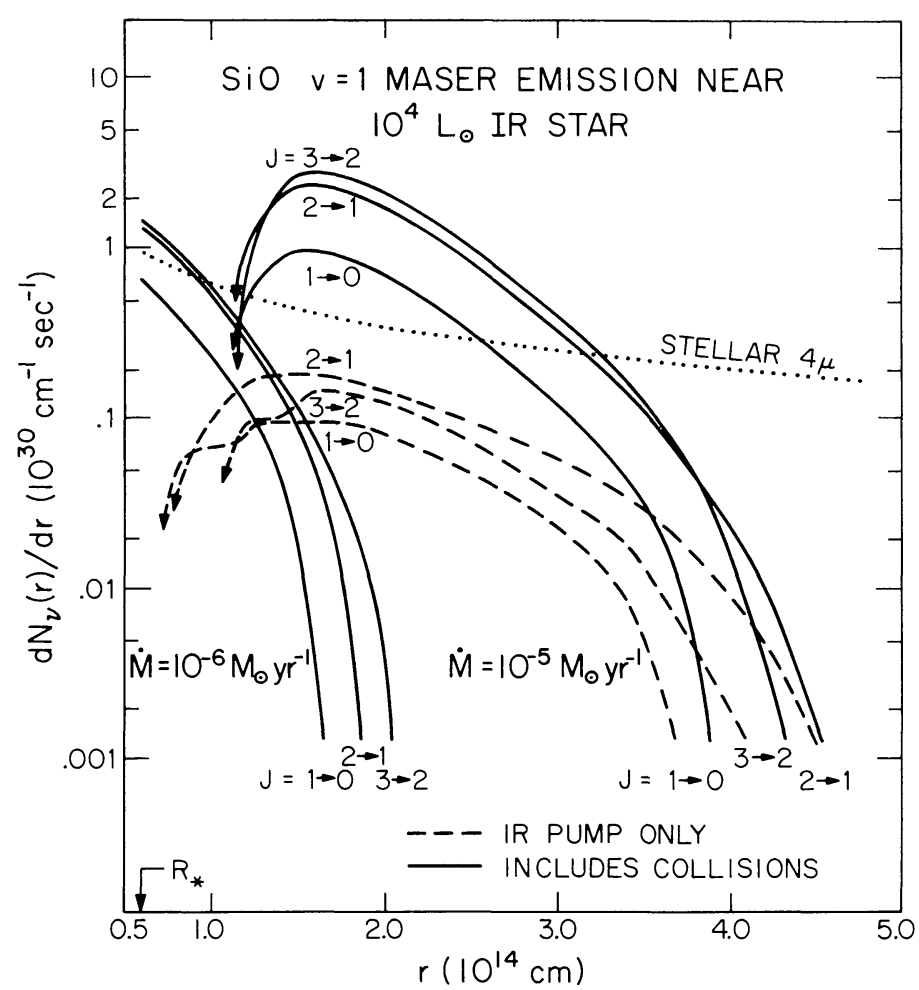

Frg. 1.-Graphs illustrating the radial dependence of the photon emission rate in $v=1, J=1 \rightarrow 0, J=2 \rightarrow 1$, and $J=3 \rightarrow 2$, for two rates of mass loss and with or without collisional excitations.

in the low-density model, the stellar radius is reached before either effect becomes important.

A detailed comparison of this theory with the observed $\mathrm{SiO}$ maser intensities requires a knowledge of the several vibration transition strengths. This comparison will be presented in a subsequent publication.

\section{COMMENTS}

The proposed pump mechanism can be generalized to give population inversions in the low-lying rotational levels of any excited vibrational state not just of $\mathrm{SiO}$ but also of other molecules with simple rotational and vibrational structures. Whether such maser action is observable will depend primarily on the abundance of the particular molecule and its dipole moment. The $v=1, J=1 \rightarrow 0$ transition of $\mathrm{CO}$ had been looked for but was not observed (Davis et al. 1974). We offer this explanation. At those infrared stars where $\mathrm{SiO}$ masers are observed, the $\mathrm{CO}$ abundance is probably not more than 10 times the $\mathrm{SiO}$ abundance (Dolan 1965; Vardya 1966). This advantage is more than offset by the very small $\mathrm{CO}$ dipole moment which is $1 / 30$ that of $\mathrm{SiO}$. This much weaker rotational line strength is unfavorable to producing observable maser emission. CS has a dipole moment only slightly smaller than that of $\mathrm{SiO}, 2.0$ debyes to 3.1 debyes for $\mathrm{SiO}$. However, at the cool, oxygen-rich giants where $\mathrm{SiO}$ masers are observed, the abundance of $\mathrm{CS}$ is only $10^{-4}$ of $\mathrm{SiO}$
(Dolan 1965; Vardya 1966). For this reason we do not expect maser action in the excited vibrational states of $\mathrm{CS}$ to be observed where the $\mathrm{SiO}$ masers are observed, but such maser action for CS might be probable toward cool, luminous, carbon-rich stars.

We have used a value of $10^{-3}$ for the ratio of the $v=0 \rightarrow 1$ transition strength to the pure rotational transition strength of $\mathrm{SiO}$. If this ratio should be lower than $10^{-4}$, our pump model would have difficulty producing the observed maser intensities, as, in order to do so, it would require an abundance of $\mathrm{SiO}$ which, for normal cosmic abundance, leads to a high density that begins to quench the maser action by collisions.

Finally, we wish to comment that population inversions in the $v=0$, low- $J$ rotational levels are possible under certain circumstances. The inversions are possible because the opacity to spontaneous emission is different from the opacity to the stellar radiation. In general, the kinematics of the mass outflow leads to a radial opacity which is greater than the averaged opacity over all directions. A situation can occur in which the radial opacity is greater than unity but the averaged opacity is less. In this case the depopulation of a $v=0$ level depends on the number of radiative excitation routes whereas the subsequent return of the population does not, and maser action in $v=0$ is then possible. The percentage of the inversion is small, however, primarily because the rate of collisions among $v=0$ levels is comparable to the 
rate of radiative depopulation. In the one model with pure radiative excitation from $v=0$ to $v=1$, we find inversion of the $v=0, J=1 \rightarrow 0$ line occurring between $5 \times 10^{14}$ and $4 \times 10^{15} \mathrm{~cm}$. The maximum number of gain lengths does not exceed 8 . The photon emission rate is at least 100 times less than that of the $v=1, J=1 \rightarrow 0$ line.
We thank Drs. P. Goldreich and P. Solomon for very helpful discussions. We also thank Dr. Roger Thompson for informing us that the CO $V=0 \rightarrow 1$ lines have Einstein $B$ coefficients of absorption that are comparable to those of $\mathrm{SiO} V .=0 \rightarrow 1$ lines.

\section{REFERENCES}

Castor, J. I. 1970, M.N.R.A.S., 149, 111.

Davis, J. H., Blair, G. N., Van Till, H., and Thaddeus, P. 1974, $A$ p.J. (Letters), 190, L117.

Dolan, J. F. 1965, A p. J., 142, 1621.

Elitzur, M., Goldreich, P., and Scoville, N. Z. 1974, to be published.
Goldsmith, P. F. 1972, $A$ p. $J ., 176,597$.

Kwok, S., Gilman, R. C., Woolf, N. J. 1974, Ap.J. (Letters), to be published.

Lucy, L. B. 1971, A p. J., 163, 95.

Snyder, L. E., and Buhl, D. 1974, Ap.J. (Letters), 189, L31.

Vardya, M. S. 1966, M.N.R.A.S., 134, 347.

JoHn KwaN: Institute for Advanced Study, Princeton, NJ 08540

N. Z. Scoville: Department of Astronomy, California Institute of Technology, Pasadena, CA 91109 